\title{
Análise numérica da influência de parâmetros operacionais e construtivos no funcionamento de trocadores de calor solo-ar
}

Numerical analysis of the influence of operational and constructive parameters on the operation of earth-air heat exchangers

\author{
C. Marzarotto ${ }^{1}$; B. Nunes ${ }^{2}$; M. K. Rodrigues ${ }^{2}$; J. A. Souza ${ }^{1,2}$; L. A. O. Rocha ${ }^{2,3}$; \\ R. S. Brum ${ }^{4}$; E. D. dos Santos ${ }^{1,2} ;$ L. A. Isoldi ${ }^{1,2}$ \\ ${ }^{1}$ Escola de Engenharia (EE), Universidade Federal do Rio Grande (FURG), 96203-900, Rio Grande-RS, Brasil \\ ${ }^{2}$ Programa de Pós-Graduação em Modelagem Computacional (PPGMC), Universidade Federal do Rio Grande \\ (FURG), 96203-900, Rio Grande-RS, Brasil \\ ${ }^{3}$ Departamento de Engenharia Mecânica (DEMEC), Universidade Federal do Rio Grande do Sul (UFRGS), 90.050- \\ 170,Porto Alegre-RS, Brasil \\ ${ }^{4}$ Departamento de Matemática e Estatística, Universidade Federal de Pelotas, 96010-900, Capão do Leão-RS, Brasil
}

marzarotto.carine@gmail.com

(Recebido em 12 de setembro de 2014; aceito em 04 de janeiro de 2015)

\begin{abstract}
A busca pelo conforto térmico em ambientes construídos eleva o consumo de energia elétrica, devido à necessidade de climatização para se atingir uma temperatura agradável: resfriamento no verão e aquecimento no inverno. A fim de reduzir o consumo de energia elétrica nos condicionadores de ar, surge o trocador de calor solo-ar (TCSA), que utiliza a radiação solar que incide sobre a superfície do solo como uma fonte de energia limpa e renovável. Assim, o ar que escoa no TCSA é direcionado para o interior de edificações, proporcionando uma melhoria significativa na sua condição térmica. Isso é possível devido à inércia térmica do solo, que possui temperatura mais amena se comparada à temperatura do ar ambiente. Dessa forma, o objetivo deste trabalho é avaliar numericamente a influência da velocidade do ar em escoamento no duto e do espaçamento entre dois dutos em paralelo no desempenho térmico do TCSA. Para isso um modelo computacional, verificado e validado, desenvolvido no software FLUENT, que é baseado no Método dos Volumes Finitos (MVF), foi usado. Os resultados mostram que durante os períodos mais quentes a menor velocidade de escoamento do ar analisada conduz a um melhor desempenho térmico do TCSA. Também foi observado que a partir de $1 \mathrm{~m}$ de afastamento entre os dutos, a troca térmica entre cada duto e solo não é afetada de forma significativa.
\end{abstract}

Palavras-chave: trocadores de calor solo-ar (TCSA), variação da velocidade de escoamento do ar, estudo do espaçamento entre dutos paralelos.

The search for thermal comfort elevates the electric energy consumption, due the need of climatization to reach a pleasurable temperature: cooling at summer and heating at winter. Looking for the reduction of electric energy consumption by air conditioners, we reach the earth-air heat exchanger (EAHE), that uses the incident solar radiation over the surface of the soil as a source of clean and renewable energy. That way the flowing air of EAHE is directed toward edifications, providing a significant improvement of the thermal condition. This is possible due to the thermal inertia of the soil, which has milder temperatures compared to the air's temperature. Thus, the goal of this study is numerically evaluate the influence of the air's flow velocity in the duct and the spacing between the parallel ducts on the thermal performance of the TCSA. For that a computational model, verified and validated, developed in the software FLUENT, which is based on the finite volume method (MVF), was employed. The results show that during the hottest periods the lowest flow velocity imposes in a better thermal performance of the TCSA. It was also observed that after $1 \mathrm{~m}$ of spacing between ducts the thermal exchange between each duct and soil is not significantly affected.

Keywords: earth-air heat exchanger (EAHE), velocity variation of air flowing, study of spacing between parallel ducts. 


\section{INTRODUÇÃO}

Os avanços científicos e tecnológicos buscam melhorar a qualidade de vida humana. A energia é fundamental neste processo evolutivo, tornando necessário buscar fontes mais sustentáveis para substituir os combustíveis fósseis e atenuar o impacto ambiental. Dessa forma, as fontes energéticas renováveis e limpas se destacam na manutenção dos recursos naturais, além de auxiliar na crescente demanda mundial por geração de energia. Talvez a mais importante das fontes de energia renovável seja o sol, por ser uma fonte inesgotável de luz e calor.

Nesse contexto a energia térmica armazenada nas camadas superficiais do solo, que é proveniente da incidência da radiação solar na crosta terrestre, pode ser aproveitada para melhorar a condição térmica de ambientes construídos proporcionando uma redução no consumo de energia elétrica necessária para o funcionamento de equipamentos de condicionamento de ar. Para isso, é possível empregar um dispositivo chamado trocador de calor solo-ar (TCSA), que tem como princípio de funcionamento o escoamento do ar ambiente externo através de dutos enterrados no solo. Como mostra a Figura 1, durante o escoamento o ar troca calor com o solo saindo do TCSA com uma temperatura mais amena [1].
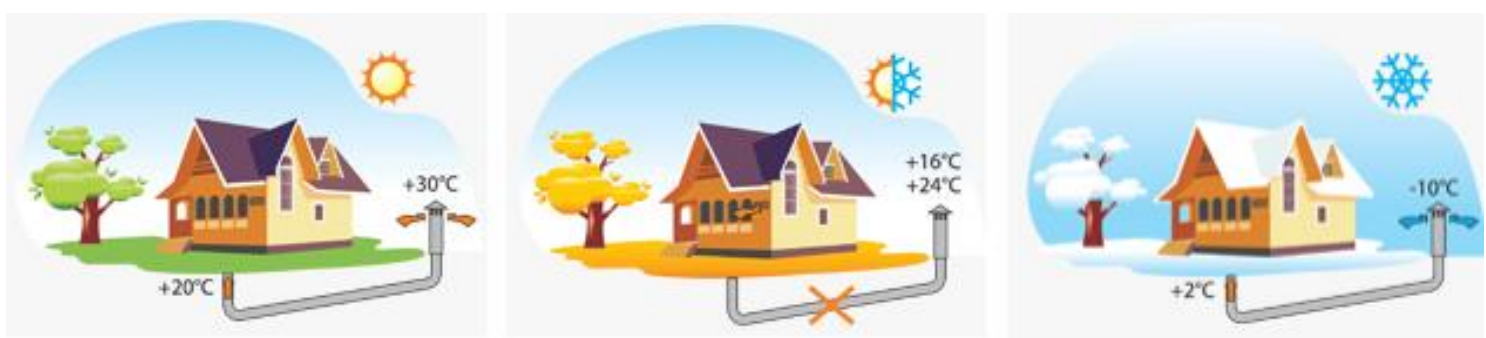

Figura 1: Ilustração do funcionamento de um TCSA.

Fonte: http://vents.ua/cat/energy-saving-geo/

Em Peretti et al. [2] foi realizada uma pesquisa bibliográfica a fim de analisar o princípio de funcionamento do TCSA, a forma da instalação, as características e condições de funcionamento, os mecanismos de transferência de calor e mecânica dos fluidos que integram o funcionamento do TCSA e suas potencialidades na melhoria térmica de ambientes construídos. Esse trabalho permite estabelecer comparações da modelagem numérica de TCSA, além de fornecer subsídio para maior compreensão e possibilidades de pesquisa sobre o assunto.

Em Trzaski e Zawada [3] é apresentada uma metodologia desenvolvida para avaliação do desempenho de TCSA. Para isso, um modelo computacional tridimensional validado e verificado foi utilizado nas simulações numéricas. Os resultados indicam que o desempenho energético do TCSA depende de uma série de parâmetros, incluindo configuração geométrica, parâmetros operacionais e fatores ambientais. Os resultados das simulações indicam que os parâmetros analisados afetam significativamente a eficiência térmica do TCSA, como o comprimento dos dutos, número de dutos em paralelos, propriedades termofísicas dos materiais, área de sombreamento do solo e de cobertura da superfície do solo.

Conforme Paepe e Janssens [4] foram realizadas, por meio de métodos analíticos unidimensionais, análises da eficiência térmica de TCSA, com relação às suas dimensões, independente do solo e das condições climáticas. Nesse trabalho foi observado que o TCSA depende de três parâmetros fundamentais: comprimento, diâmetro e número de dutos paralelos. Além disso, foi notado que o desempenho térmico é melhorado com a redução do diâmetro e aumento do número de dutos em paralelo.

Assim, o objetivo do presente trabalho é avaliar numericamente a influência de um parâmetro de funcionamento, a velocidade de escoamento do ar, e de um parâmetro construtivo, o espaçamento mínimo entre dois dutos paralelos, no comportamento térmico de TCSA. Para isso foi empregado um modelo computacional tridimensional verificado e validado [5], desenvolvido no software FLUENT que é baseado no Método dos Volumes Finitos (MVF). Na análise envolvendo a velocidade de escoamento do ar no TCSA foram consideradas cinco diferentes magnitudes para a mesma, permitindo avaliar como esse parâmetro afeta a 
performance do dispositivo. Já a investigação relativa ao espaçamento entre dutos paralelos visa definir qual a distância mínima para a instalação dos mesmos de maneira que a presença de um duto não interfira significativamente no desempenho térmico do outro duto. Então, para avaliar o efeito desses parâmetros no desempenho térmico do TCSA, a temperatura de saída do ar foi considerada. Tais parâmetros operacionais e construtivos definem o desempenho térmico destas instalações.

\section{MATERIAL E MÉTODOS}

Nesta seção será apresentada a modelagem matemática e numérica para a abordagem computacional para aplicações em TCSA. Neste estudo foi utilizado o modelo simplificado de Brum et al. [5] validado e verificado com base em Vaz et al. [6].

\subsection{MATERIAL E MÉTODOS}

Os modelos matemáticos utilizados para a abordagem computacional de TCSA são fundamentados no princípio de conservação da massa, quantidade de movimento e energia, além da modelagem da turbulência [7]. Dessa forma, de acordo com Patankar [8] e Maliska [9], a equação de conservação da massa ou equação da continuidade, pode ser escrita na sua forma simplificada como:

$$
\nabla \cdot(\vec{v})=0
$$

onde: $\vec{v}$ é vetor velocidade do escoamento $(\mathrm{m} / \mathrm{s})$ e $\nabla$ é o operador diferencial, que em coordenadas retangulares, é dado por: $\nabla=\vec{i} \frac{\partial}{\partial x}+\vec{j} \frac{\partial}{\partial x}+\vec{k} \frac{\partial}{\partial x}$, sendo: $\vec{i}, \vec{j}, \vec{k}$ os vetores unitários direcionais que são base para o espaço $\mathrm{R}^{3}$ (espaço tridimensional). Dessa forma, a Equação (1) assume a condição de escoamento incompressível e sem fontes de massa [9].

Segundo Maliska [9], a equação da conservação da quantidade de movimento é dada por:

$$
\frac{\partial \rho \overline{\vec{v}}}{\partial t}+\nabla \cdot(\rho \overline{\vec{v} \vec{v}})=-\nabla \bar{p}+\nabla \cdot \overline{(\overline{\bar{\tau}})}
$$

onde: $\rho$ é a massa específica do $\operatorname{ar}\left(\mathrm{kg} / \mathrm{m}^{3}\right) ; p$ é a pressão estática (piezométrica) do fluido $\left(\mathrm{N} / \mathrm{m}^{2}\right) ; \overline{\bar{\tau}}$ é o tensor de tensões viscosas relacionadas às forças de superfície em um elemento infinitesimal de fluido $\left(\mathrm{N} / \mathrm{m}^{2}\right)$.

Tendo em conta que o escoamento é incompressível, o tensor de tensões viscosas é representado por:

$$
\bar{\tau}=\mu\left[\left(\nabla \vec{v}+\nabla \vec{v}^{T}\right)\right]
$$

A equação da conservação da energia, na forma mais geral, com base em [9], pode ser expressa por:

$$
\frac{\partial \rho h}{\partial t}+\nabla[\vec{v}(\rho h)]=\nabla \cdot\left[k_{e f f} \nabla T\right]
$$

A entalpia específica, representada pela variável $h$, pode ser expressa por:

$$
h=\int_{T_{r e f}}^{T} C_{p} d T
$$


onde: $C_{p}$ é o calor específico à pressão constante $(\mathrm{J} / \mathrm{kgK}), T$ é a temperatura $(\mathrm{K})$ e $T_{r e f}$ é uma temperatura em um estado termodinâmico de referência $\left(T_{r e f}=298,15 \mathrm{~K}\right)$. Por fim, a condutividade efetiva, ou seja, $k_{e f f}$, $(\mathrm{W} / \mathrm{m} \cdot \mathrm{K})$, é dada por $k+k t$, onde $k$ é a condutividade térmica e $k t$ é a condutividade térmica turbulenta, definida em concordância com o modelo de turbulência adotado.

Para a região do solo, foi resolvida a equação da difusão do calor, que pode ser expressa por:

$$
\nabla^{2} T=\frac{1}{\propto} \frac{\partial T}{\partial t}
$$

onde: $\propto$ é a difusividade térmica no solo, sendo igual a $65,54 \cdot 10^{-8} \mathrm{~m}^{2} / \mathrm{s}$, e $\nabla^{2}$ é o operador de Laplace, que é definido como: $\nabla^{2}=\left(\frac{\partial^{2} T}{\partial x^{2}}+\frac{\partial^{2} T}{\partial y^{2}}+\frac{\partial^{2} T}{\partial z^{2}}\right)$, para o sistema de coordenadas retangulares.

Neste trabalho, o modelo de turbulência adotado foi o $k-\varepsilon$. Em Kipeer [10], o modelo $k-\varepsilon$ foi utilizado satisfatoriamente em aplicações de TCSA.

O modelo $k$ - $\varepsilon$ é um modelo de duas equações: a energia cinética $(k)$ e a dissipação viscosa turbulenta $(\varepsilon)$. Atualmente, este é o modelo mais empregado e considerado em estudos científicos e em aplicações tecnológicas.

Este modelo de turbulência exibe soluções rápidas computacionalmente para vários problemas da engenharia. De acordo com Versteeg e Malalasekera [11], o modelo apresenta problemas para caracterizar escoamentos rotacionais, com linhas de correntes curvas, separações e regiões estagnadas. E para melhorar os resultados, nestes casos, é necessário ajustar as constantes do modelo.

\subsection{MODELAGEM NÚMERICA}

A utilização da simulação numérica para a análise dos escoamentos de fluidos, transferência de calor e demais fenômenos associados é conhecida como Dinâmica dos Fluidos Computacional (CFD - Computational Fluid Dynamics). Quando se faz o uso da CFD obtém-se algumas vantagens, como por exemplo uma redução significativa de tempo e de custos em novos projetos, capacidade de estudar sistemas onde as análises experimentais são complexas e um nível alto de detalhes nos resultados [11].

No pré-processamento são realizadas: a definição do problema físico, a imposição das condições iniciais e de contorno do problema, a definição do domínio computacional e a geração da malha. Nessa fase os softwares GAMBIT e FLUENT foram empregados.

No processamento, realiza-se a solução do problema. Existem vários métodos de discretização para as equações diferenciais. O software FLUENT usa o Método dos Volumes Finitos (MVF), que é caracterizado pela obtenção de equações aproximadas por meio da resolução de balanços de massa, energia e quantidade de movimento em um determinado volume de controle sobre um meio contínuo. A interpretação física direta das equações resultantes da aplicação do método e a possibilidade de aplicá-lo sobre as malhas com espaçamentos não-uniformes, são as duas razões que explicam a aderência ao emprego do método [11].

Para os termos advectivos foi utilizado o esquema de advecção upwind. De acordo com Dos Santos [12], a direção do escoamento, neste esquema, é levada em consideração para a realização do cálculo do valor de uma determinada variável, como velocidade ou temperatura na face do volume. Além disso, sempre é garantida a positividade dos coeficientes dos termos advectivos, sendo geradas soluções numéricas ausentes de oscilações numéricas.

Para o acoplamento pressão-velocidade foi utilizado o modelo de solução acoplada Coupled. Trata-se de um modelo robusto, pois possui um desempenho melhor ao ser comparado com algoritmos de solução segregados. 
Por fim, no pós-processamento, os resultados são apresentados e analisados. Os pacotes de CFD, nesta etapa, estão equipados com ferramentas gráficas capazes de auxiliar na visualização dos resultados.

\subsection{ABORDAGEM COMPUTACIONAL}

Dos estudos de Brum et al. [5] e Vaz et al. [6], retirou-se as dimensões do domínio computacional, que é composto por uma porção de solo onde estão inseridos um ou mais dutos representando o TCSA. Considerou-se um solo argiloso e para a discretização dos domínios computacionais foram utilizados volumes finitos tetraédricos.

No GAMBIT foram criadas as geometrias para representar a instalação do TCSA e o solo com as dimensões adequadas para cada caso. Os dutos foram representados com um diâmetro de $110 \mathrm{~mm}$, instalados a uma profundidade de $3 \mathrm{~m} \mathrm{[5]} \mathrm{e} \mathrm{a} \mathrm{espessura} \mathrm{de} \mathrm{suas} \mathrm{paredes} \mathrm{não} \mathrm{foi}$ levada em consideração [6].

Estabeleceram-se as condições de contorno para a entrada do duto como velocity inlet (condições do ar na entrada do duto), para a saída do duto como velocity outlet (condição do ar na saída do duto), na superfície do solo como wall (variação térmica na superfície) e as demais superfícies do solo foram consideradas isoladas termicamente. Identificaram-se os tipos de materiais utilizados no domínio computacional, "sólido" para o solo e "fluido" para o ar dos dutos. Ao fim desta etapa, as malhas foram geradas e exportadas para o FLUENT.

Em cada uma das simulações apresentadas nesse trabalho foi considerado um intervalo de tempo de 2 anos. Onde o primeiro ano de simulação se faz necessário para estabilizar a temperatura do solo, e no segundo ano de simulação as análises necessárias foram realizadas. Utilizou-se um passo de tempo de 3600 s, com um máximo de 200 iterações para cada passo de tempo.

A Tabela 1 apresenta as propriedades termofísicas adotadas para o solo e para o ar [6].

Tabela 1: Propriedade dos Materiais.

\begin{tabular}{cccc}
\hline & \multicolumn{3}{c}{ Propriedade dos materiais. } \\
\cline { 2 - 4 } Componentes & $\rho\left(\mathbf{k g} / \mathbf{m}^{3}\right)$ & $k(\mathbf{W} / \mathbf{m} \cdot \mathbf{K})$ & $c_{p}(\mathbf{J} / \mathbf{k g} \cdot \mathbf{K})$ \\
\hline Solo & 1.800 & 2,1 & 1.780 \\
$\mathbf{A r}$ & 1,16 & 0,0242 & 1.010 \\
\hline
\end{tabular}

Em seguida foram definidas as condições de contorno necessárias para a solução do problema: velocidade prescrita do ar na entrada no duto, temperatura prescrita do ar na entrada do duto em função do tempo, pressão atmosférica prescrita na saída do duto e temperatura prescrita na superfície superior do solo em função do tempo. A variação anual de temperatura na entrada do ar no duto e a variação anual de temperatura da superfície do solo são representadas, respectivamente, por Vaz [1] e Brum et al. [5]:

$$
\begin{aligned}
& T_{e}(t)=296,18+6,92 \cdot \operatorname{sen}\left(200 \cdot 10^{-9} \cdot t+26,42\right) \\
& T_{S}(t)=291,70+6,28 \cdot \operatorname{sen}\left(200 \cdot 10^{-9} \cdot t+26,42\right)
\end{aligned}
$$

onde: $T_{e}(t)$ é a temperatura do ar na entrada duto ao longo do tempo $(\mathrm{K}), T_{s}(t)$ é a temperatura na superfície do solo em função do tempo (K) e $t$ é o tempo (s).

As Equações (7) e (8) são funções periódicas ajustadas estatisticamente a partir de dados experimentais de [1]. Essas funções foram inseridas no FLUENT na forma de Função Definida pelo Usuário (UDF), que é um algoritmo em linguagem $\mathrm{C}$, que ao ser compilado permite que estas funções sejam utilizadas como condições de contorno do problema [7].

Para a análise dos resultados, a temperatura de saída do ar do duto foi monitorada, permitindo comparar os resultados com os apresentados em Brum et al. [5], verificando o 
modelo numérico empregado no presente trabalho, para um caso com o único duto e com velocidade prescrita do ar na entrada do duto de $3,3 \mathrm{~m} / \mathrm{s}$.

\section{RESULTADOS E DISCUSSÃO}

Para as análises numéricas foram utilizadas duas configurações para o TCSA (Figura 2), ambas instaladas a uma profundidade de $3 \mathrm{~m}$ [5]. Na Instalação 1 (Figura 2a) o TCSA consiste de um duto com diâmetro $d=110 \mathrm{~mm}$, sendo usada para avaliar a influência da velocidade do escoamento do ar em seu comportamento térmico. Considerando Rodrigues [7], foram gerados 39.392 volumes finitos tetraédricos no duto (tamanho máximo $d / 3$ ) e 575.852 volumes finitos tetraédricos no solo (tamanho máximo 3d), totalizando uma malha com 615.244 células.

Já para o estudo do espaçamento mínimo entre dutos, um TCSA composto por dois dutos paralelos com o mesmo diâmetro $d=110 \mathrm{~mm}$ instalados em uma profundidade de $3 \mathrm{~m}$ (Figura $2 b$ ) foi utilizado. O espaçamento horizontal $\left(S_{h}\right)$ entre os dutos foi variado, a fim de avaliar a influência desse parâmetro no comportamento térmico do TCSA. Para essa análise, considerando Brum et al. [5], Vaz et al. [6] e Rodrigues [7], a velocidade do ar prescrita na entrada do TCSA foi de 3,3 m/s para todos os espaçamentos numericamente simulados.

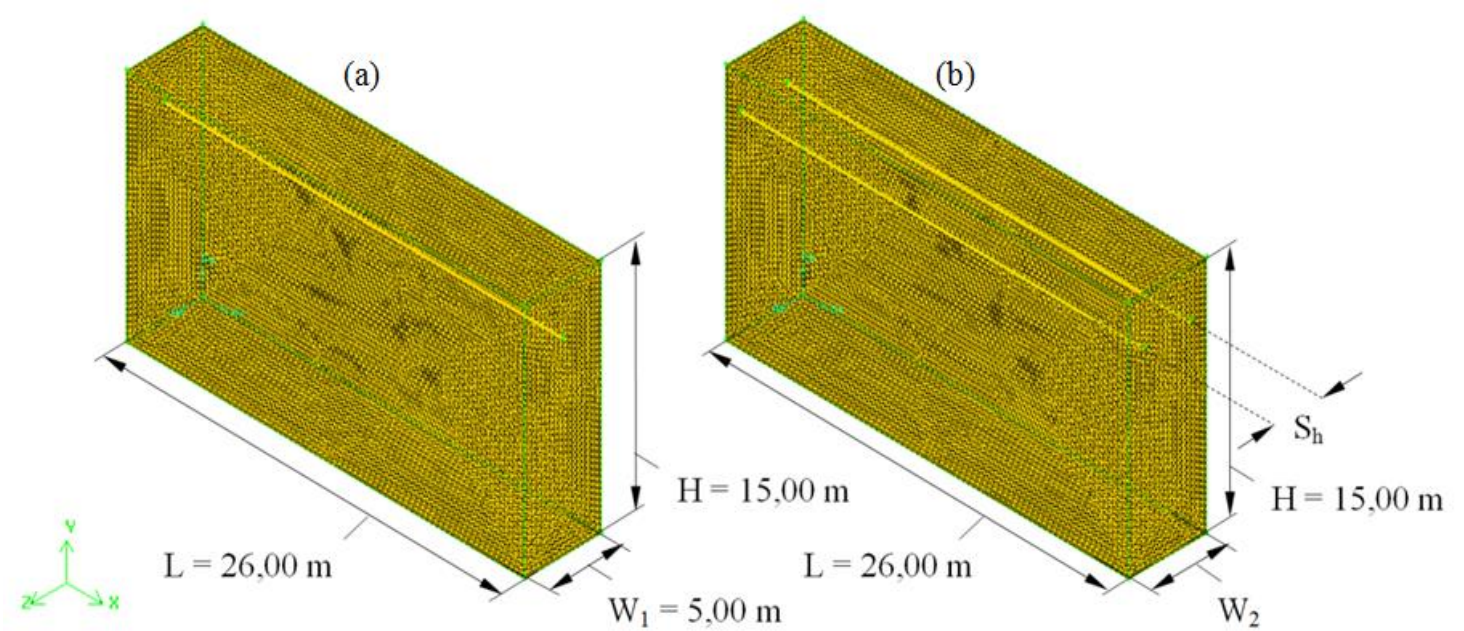

Figura 2: Domínio computacional (sem escala): (a)Instalação 1 e (b)Instalação 2.

Devido à variação do valor de $S_{h}$ a largura da porção de solo $W_{2}$ no domínio computacional também variou (Figura 2b). Esses valores, bem como o número de volumes finitos tetraédricos gerados estão apresentados na Tabela 2. Em cada caso manteve-se uma distância de 5,00 m entre os dutos e as paredes laterais do solo, a fim de se evitar a influência das condições de contorno de fluxo prescrito nulo (das superfícies laterais) nos resultados.

Cabe destacar que o tamanho máximo para as células computacionais nos dutos e no solo seguem o mesmo critério usado na discretização da Instalação 1. 
Tabela 2: Volumes finitos tetraédricos nas malhas geradas.

\begin{tabular}{lcccccc}
\hline Caso & $\begin{array}{c}\text { Espaçamento } \\
\text { horizontal } \boldsymbol{S}_{\boldsymbol{h}}(\mathbf{m})\end{array}$ & $\begin{array}{c}\text { Largura } \\
\boldsymbol{W}_{\mathbf{2}}(\mathbf{m})\end{array}$ & Solo & Duto 1 & Duto 2 & $\begin{array}{c}\text { Total da } \\
\text { Malha }\end{array}$ \\
\hline Caso 1 & 0,25 & 10,25 & 1.163 .190 & 39.392 & 39.392 & 1.241 .974 \\
Caso 2 & 0,50 & 10,50 & 1.185 .352 & 39.392 & 39.392 & 1.264 .136 \\
Caso 3 & 0,75 & 10,75 & 1.197 .857 & 39.392 & 39.392 & 1.276 .641 \\
Caso 4 & 1,00 & 11,00 & 1.259 .868 & 39.392 & 39.392 & 1.338 .652 \\
Caso 5 & 2,00 & 12,00 & 1.272 .691 & 39.392 & 39.392 & 1.351 .475 \\
Caso 6 & 4,00 & 14,00 & 1.415 .467 & 39.392 & 39.392 & 1.494 .251 \\
Caso 7 & 6,00 & 16,00 & 1.479 .843 & 39.392 & 39.392 & 1.558 .627 \\
Caso 8 & 8,00 & 18,00 & 1.862 .354 & 39.392 & 39.392 & 1.941 .138 \\
Caso 9 & 10,00 & 20,00 & 1.973 .775 & 39.392 & 39.392 & 2.052 .559 \\
\hline
\end{tabular}

\subsection{ESTUDO DA VARIAÇÃO DA VELOCIDADE DO ESCOAMENTO DO AR}

Foram adotadas cinco magnitudes para a velocidade de escoamento do ar no TCSA: $1 \mathrm{~m} / \mathrm{s}$, $2 \mathrm{~m} / \mathrm{s}, 3 \mathrm{~m} / \mathrm{s}, 4 \mathrm{~m} / \mathrm{s}$ e $5 \mathrm{~m} / \mathrm{s}$. Para avaliar como a velocidade de escoamento afeta o comportamento térmico do TCSA, sua temperatura de saída foi monitorada e os resultados para um ciclo anual estão apresentados na Figura 3.

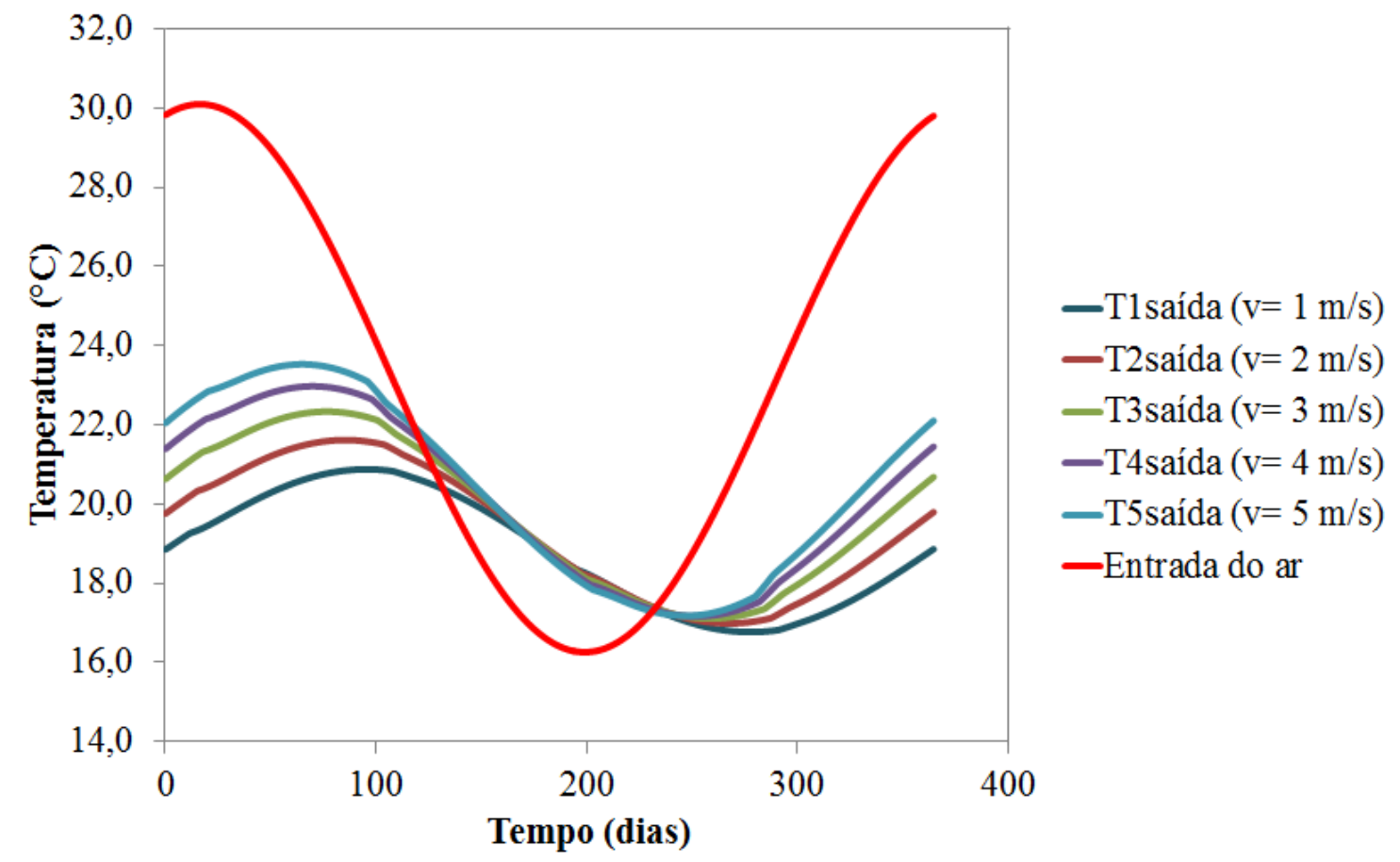

Figura 3: Variação anual da temperatura de saída do TCSA para diferentes velocidades de escoamento do ar.

Tomando como referência o dia 0 (que representa o primeiro dia do ano), é possível notar na Figura 3 que durante os meses de verão a eficiência do TCSA é superior se comparada à do inverno, ou seja, o ar que sai do TCSA no verão apresenta uma variação de temperatura maior em relação à temperatura do ar que entra no TCSA, estando esse comportamento térmico de acordo com estudos anteriores [5, 7]. Pode-se perceber também que as velocidades de escoamento menores permitem uma melhoria no desempenho do TCSA no verão. Isso é comprovado, por exemplo, no primeiro dia do ano: com a menor velocidade de $1 \mathrm{~m} / \mathrm{s}$ foi obtida 
uma diminuição da temperatura do ar de aproximadamente $11^{\circ} \mathrm{C}$, enquanto que para a maior velocidade de $5 \mathrm{~m} / \mathrm{s}$ a diminuição da temperatura foi em torno de $8{ }^{\circ} \mathrm{C}$. Portanto, a velocidade de $1 \mathrm{~m} / \mathrm{s}$ permite um ganho de aproximadamente $3{ }^{\circ} \mathrm{C}$ em relação à velocidade de $5 \mathrm{~m} / \mathrm{s}$ para os dias de maior calor. Para meses de inverno, observa-se que o desempenho do TCSA é praticamente o mesmo para todas as velocidades estudadas. Além de o desempenho térmico ser inferior ao obtido para os meses quentes, porque a variação da temperatura de entrada e saída do duto é pouco satisfatória.

\subsection{ESTUDO DO ESPAÇAMENTO MÍNIMO ENTRE DUTOS PARALELOS}

Na Figura 4, a partir da variação do espaçamento $S_{h}$ dos dutos (Tabela 2) e considerando a temperatura de saída do TCSA, também monitorada durante um ciclo anual, foi possível avaliar como esse parâmetro influencia o comportamento térmico do dispositivo. É importante destacar que o resultado para a Instalação 1 (Figura 2a), considerando uma velocidade de escoamento de $3,3 \mathrm{~m} / \mathrm{s}$, foi usado como referência e, para isso, também foi apresentado na Figura 4.

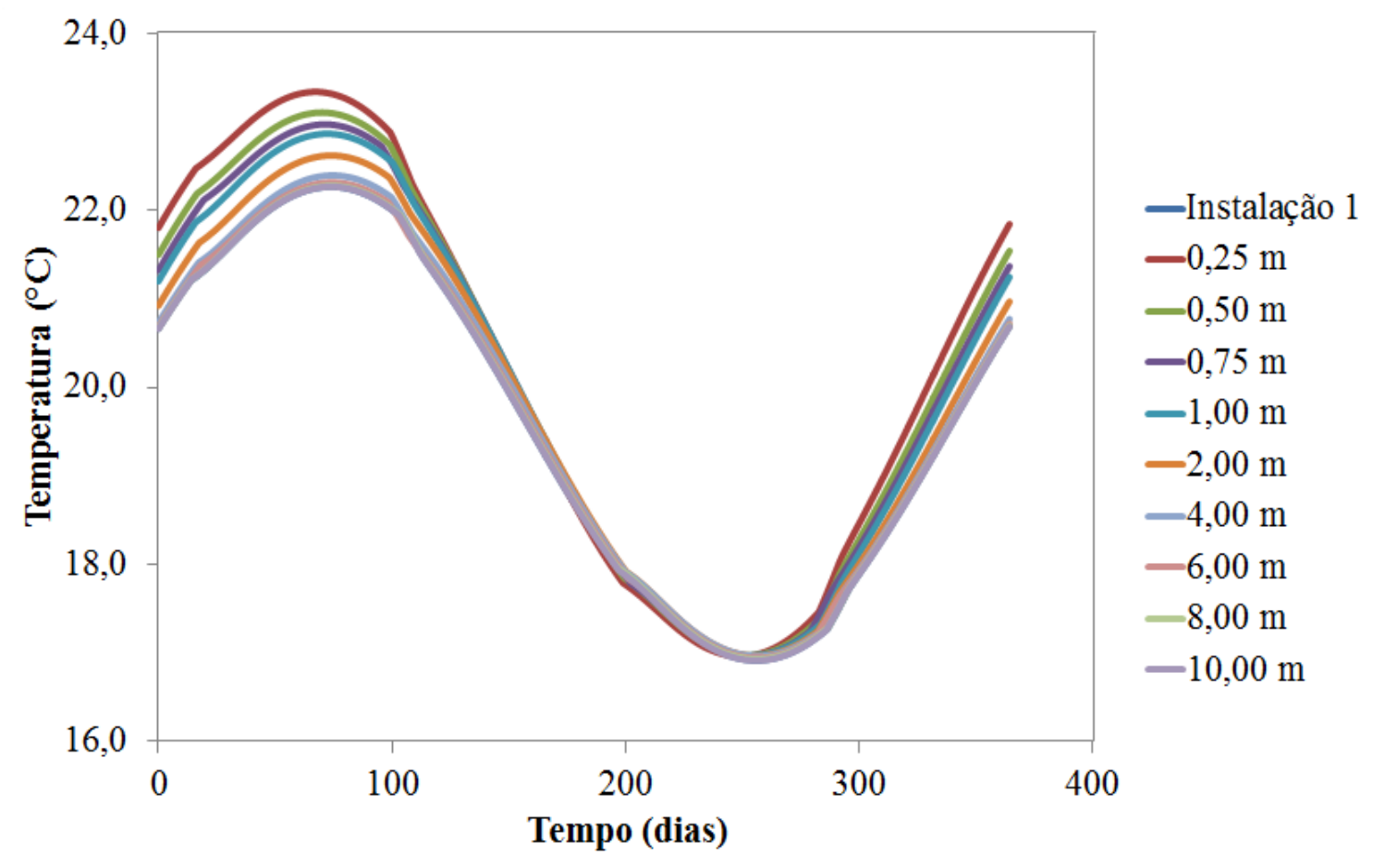

Figura 4: Variação anual da temperatura de saída do ar do TCSA para diferentes valores de espaçamento $S_{h}$.

Como já era esperado, à medida que o afastamento $S_{h}$ entre dutos aumenta, o comportamento térmico do TCSA com dois dutos (Instalação 2) se aproxima ao comportamento térmico do TCSA com um duto (Instalação 1). Para exemplificar essa tendência, na Figura 5 é apresentada a temperatura média na saída do TCSA para o último dia do ano para os diversos espaçamentos avaliados. 


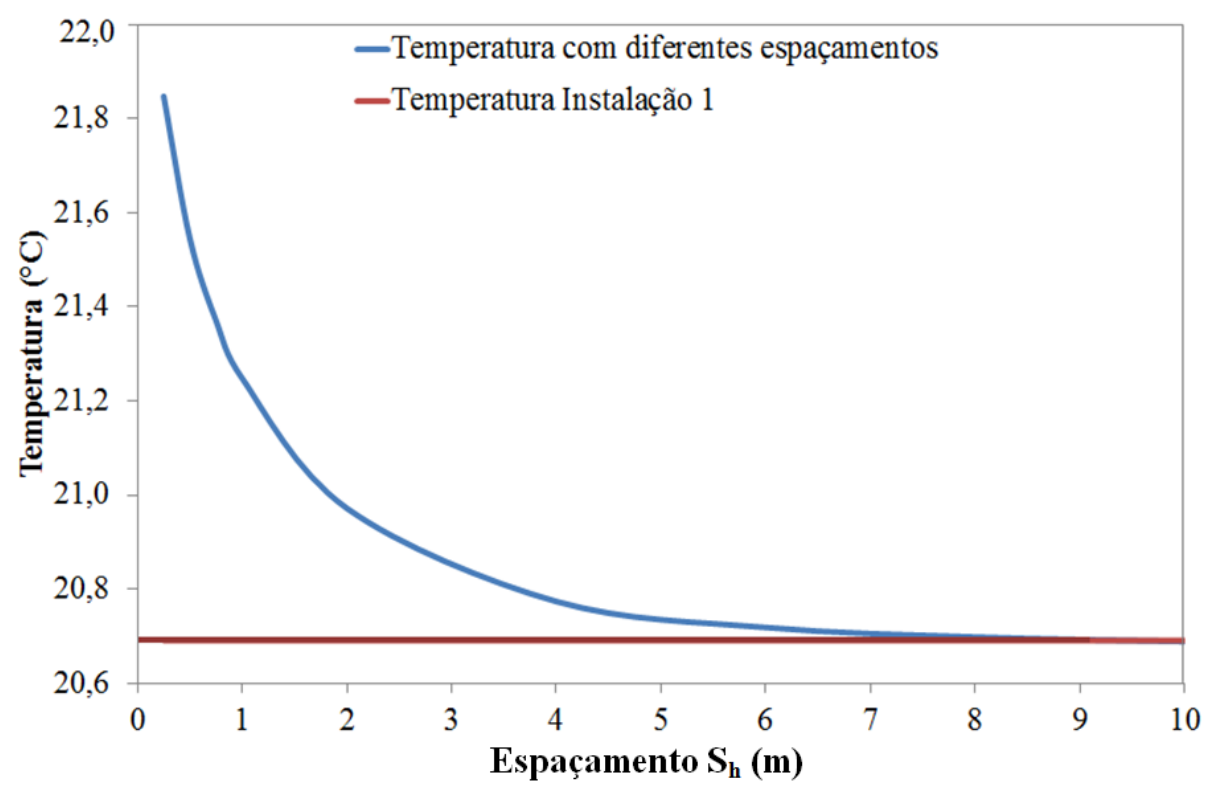

Figura 5: Temperatura de saída para o último dia de simulação com diferentes afastamentos entre dutos.

Figura 5 fica claro que para um TCSA com dois dutos apresentar um comportamento térmico idêntico a um TCSA com um duto, ou seja, que não haja interferência na troca térmica de um duto no outro duto é necessário um espaçamento entre dutos de pelo menos $10 \mathrm{~m}$.

Entretanto, para quantificar essa diferença, na Tabela 3 é apresentada a diferença relativa entre os resultados médios de temperatura na saída do TCSA da Instalação 2 para os diferentes valores de $S_{h}$ em relação ao resultado da Instalação 1.

Tabela 3: Variação percentual da temperatura de saída do ar entre a Instalação 2 e a Instalação 1.

\begin{tabular}{cc}
\hline & $\begin{array}{c}\text { Variação da temperatura } \\
\text { Espaçamento } \boldsymbol{S}_{\boldsymbol{h}} \text { entre dutos }(\mathbf{m})\end{array}$ \\
\cline { 2 - 2 } & $\begin{array}{c}\text { Variação da temperatura em relação a } \\
\text { Instalação } \mathbf{1}(\boldsymbol{\%})\end{array}$ \\
\hline 0,25 & 5,580 \\
0,50 & 4,120 \\
0,75 & 3,280 \\
1,00 & 2,690 \\
2,00 & 1,350 \\
4,00 & 0,400 \\
6,00 & 0,140 \\
8,00 & 0,040 \\
10,00 & 0,005 \\
\hline
\end{tabular}

\section{CONCLUSÃO}

A utilização de um TCSA permite a melhoria da condição térmica de ambientes construídos, causando uma diminuição significativa no uso de equipamentos convencionais de condicionamento de ar, proporcionado uma redução no consumo de energia elétrica para obter um ambiente com temperatura agradável. Sendo assim, estudos visando melhorar o desempenho desses dispositivos são justificados. No presente trabalho um parâmetro operacional, a velocidade de escoamento do ar no TCSA, e um parâmetro construtivo, a distância entre dois trechos paralelos de duto, foram numericamente investigados.

Com o estudo de variação de velocidades de escoamento do ar foi possível concluir que durante os períodos mais quentes (meses de janeiro, fevereiro, novembro e dezembro) o TCSA 
em estudo apresenta resultados satisfatórios para melhoria da condição térmica de ambientes construídos. Já durante os meses mais frios (maio, junho, julho e agosto) a velocidade de escoamento do ar não apresenta alterações significativas para a temperatura de saída do ar no duto da instalação de TCSA estudada. Além disso, foi possível observar que velocidades de escoamento do ar no TCSA menores conduzem a uma melhor performance do equipamento, pois permitem que o ar durante o escoamento fiquem um maior tempo em contato com o solo o que permite uma maior troca térmica. Porém essa melhoria foi observada somente para os períodos quentes. Durante os períodos frios a troca térmica no TCSA não foi influenciada de maneira relevante pela velocidade de escoamento do ar.

Com o objetivo de estudar diferentes configurações geométricas para os dutos de um TCSA, onde trechos de dutos serão dispostos paralelamente, foi simulado o comportamento térmico de um TCSA com dois dutos em paralelo (Instalação 2), visando determinar um espaçamento mínimo entre os dutos em que a troca térmica em cada duto não seja afetada significativamente. Dessa forma, como já era esperado, percebeu-se que, quanto maior o afastamento entre os dutos, menor é a variação da temperatura se comparada com a temperatura de saída do ar na Instalação 1. Porém, se for considerada a questão construtiva de um TCSA com geometria complexa, é interessante que o mesmo tenha o menor espaçamento entre dutos possível, ocupando assim uma menor região de solo. Portanto, com base nos resultados obtidos, recomenda-se um afastamento de $1 \mathrm{~m}$ entre os dutos paralelos, pois a troca térmica entre eles não é afetada de forma relevante e esse é um espaçamento aceitável para a construção de um TCSA com geometrias complexa.

\section{AGRADECIMENTOS}

Os autores agradecem ao CNPq, à CAPES e à FURG pelo apoio para o desenvolvimento da pesquisa.

\section{REFERÊNCIAS BIBLIOGRÁFICAS}

1. Vaz J. Estudo experimental e numérico sobre o uso do solo como reservatório de energia para o aquecimento e resfriamento de ambientes edificados. Tese de Doutorado, Universidade Federal do Rio Grande do Sul, Programa de Pós-Graduação em Engenharia Civil (PPGEC), 2011.

2. Peretti C, Zarrella A, Carli M, Zecchin R. The design and environmental evaluation of earth-to-air heat exchangers (EAHE). A literature review. Renewable and Sustainable Energy Rev. 2013 Dec;28:107-16, doi:10.1016/j.rser.2013.07.057.

3. Trzaski A, Zawada B. The influence of environmental and geometrical factors on air-ground tube heat exchanger energy efficiency. Build Environ. 2011 Jul;46(7):1436-44, doi:10.1016/j.buildenv.2011.01.010.

4. Paepe MD, Janssens AA. Thermo-hydraulic design of earth-air heat exchangers. Energy Build. 2011 May;35(4):389-97, doi:10.1016/S0378-7788(02)00113-5.

5. Brum RS, Rocha LAO, Vaz J, dos Santos ED, Isoldi LA. Development of simplified numerical model for evaluation of the influence of soil-air heat exchanger installation depth over its thermal potential. Int J Advanced Renewable Energy Research. 2012 Nov;1(9):505-14.

6. Vaz J, Sattler MA, dos Santos ED, Isoldi LA. Experimental and numerical analysis of an earth-air heat exchanger. Energy Build. 2011 Sep;43(9):2476-82, doi:10.1016/j.enbuild.2011.06.003.

7. Rodrigues M. Modelagem Computacional Aplicada à Melhoria do Desempenho Térmico de Trocador de Calor Solo-Ar Através do Método Constructal Design. Dissertação de Mestrado, Universidade Federal do Rio Grande, Programa de Pós-Graduação em Modelagem Computacional (PPGMC), 2014.

8. Patankar S. Numerical heat transfer and fluid flow. New York: McGraw-Hill; 1980. 197 p.

9. Maliska C. Transferência de calor e mecânica dos fluidos computacional. Rio de Janeiro: Livros Técnicos e Científicos Editora S.A.; 2004. 433 p.

10. Kipeer R. Análise Numérica de Trocadores de Calor Solo-Ar para Diferentes Configurações de Instalação. Trabalho de Conclusão do curso de Engenharia Mecânica, Universidade Federal do Rio Grande (FURG), 2014.

11. Versteeg HK, Malalasekera W. An introduction to computational fluid dynamics - the finite volume method. England: Pearson; 2007. 503 p. 
12. Dos Santos E. Análise numérica de escoamentos turbulentos não reativos com transferência de calor por convecção e radiação térmica em meios participantes. Tese de Doutorado. Universidade Federal do Rio Grande do Sul (UFRGS). Programa de Pós-Graduação em Engenharia Mecânica (PROMEC), 2011. 